\title{
5 Circular thinking and linear exposition Circling around a point to discover the point
}

When we think of a circle we think of a return to a point. When we think of lines we think of queuing. Circles are confining, but lines give one a sense of liberty and of freedom. E. B. White defined democracy as standing in line. When we get in line we customarily need first to get to the end of the line. Lines give one a sense of equality. We have no special social privileges as individuals do in queuing starting from the rear of the line. We do not return to any point unless the line returns to the original point of origination.

And when we get in that line and that ends in the grave there are no special exclusionary privileges from such a fate. In thinking about thinking we also get in a line whose terminus of our thoughts are to be discovered as we move down that line. We need not necessarily know our destination unless someone has been down that line before and tells us what lies ahead. Thus linear thinking without knowing of what is ahead is lining up all of our ducks in a row on our route to discovery.

But circular thinking always revolves and then returns to the same point. It is cyclical. What is interesting about circles is the perspective we have from each and every point on the arc of a circle. It is simply a different set of orientations. With lines we go simply to the next spot ahead with only our hoped for and wished for expectations. Our perspective is always looking forward. But in circles we can surround each other with the perspectives of others.

I was listening to a discussion about sports on TV recently, and one speaker asked the other, who supposedly was a so called expert about baseball, 'Is baseball as a game different from other sports?' What struck me about this discussion was that the speakers were circling through the various ways they wanted to use their terms: 'What is a game?', 'What is a sport?', 'Is baseball truly a game?', 'Is it truly a sport?' And again, 'Are not games and sports nothing more than different forms of entertainment?', 'How is baseball just a merely a game-show or a performance, or an entertainment?' And when we are listening to the news, 'Is it really just news, or is it just a game-show, or a form of entertainment just like baseball?'

What appears to be going on in these sorts of exchanges in questioning about what is truly the case the discussion is not about making any points about facts or about what is true of the world in any absolute sense. Rather the participants are circling through each other's vocabulary using their preferred uses of words such as game and sport in the contexts in which they prefer to use them so as to make their own judgments about social value and political practicality. In sum they are talking about how they are using words and then in turn using their preferred uses of their terms to make judgments or appeals to others to make policy judgments using the senses of terms they use to make appeals to their audiences. 
It has struck me that each individual has his or her personal language that differs from the language of other individuals. The major problem in communication is to interpret the meaning of each other's own personal language. Each person's language is very much like the development of each other's DNA. It consists of fragments of our ancestors' DNA. So it is the case that our personal language consists of fragment of meanings derived from the interpretations of persons we encounter who speak the same cultural language we speak. Language thus is anchored in the individual language we learn and use as we develop the personal and individual language we speak that is an amalgam of many different speakers.

Note all the nonsense spouted about who is a liberal, a socialist, a racist, conservative, or even just a Christian. All such terms haves no precise meaning in ordinary discourse. Each of these terms as used in contemporary politics have loaded or emotive senses of just how much value additive priority people want to give to each term as we personally use them in their contextual uses of them in dealing with each other. Note comparably how such questions about terms arise in political discourse about justice, equity, fairness, compensation, and what one owes another.

Such discussions of abstract terms usually involve the same sort of verbal circling 'Are you happy?', 'What is happiness?', 'Is happiness a brain state?', 'Are you happy only after the fact?', 'Is happiness to be free from misery and pain?' Such questions are about the different ways that people choose to give meaning and value priorities to the uses of such terms in the language they develop for themselves such as they learn to speak and develop new meanings for words for such things as happiness, misery, pain, pleasure, bliss, contentment, satisfaction, euphoria, being fulfilled, and goal satisfaction.

You can name the other synonyms for joy to enlarge this circle. How can you talk about life without ranking and relating to these synonyms and antonyms? How can we without thinking of them as correlatives define and distinguish how we use these terms in communicating? And importantly we need at least one term in such clusters of synonyms and antonyms to stabilize positively our meaning contrasts in our coming to terms with the others (Yoos, 2009).

What people are doing in circling through distinctions and differences between our uses of words is interpreting their own pattern of uses of them. And when we interpret them we discover the vocabulary that people want to use to talk about how they want to relate to each other, and again about how best in what terms that they should want to relate to one another. Usually in their attempted definitions and prescriptions of use, or what they might think of as usage, they are trying to give technical definitions of such terms without the social contexts and without the power and authority that legitimize standardized uses of technical terms.

They are not about seeking conclusions about what is the case, but they are discussing how they want to talk to each other about their meanings in their dealings with each other. They are seeking language to talk about the conclusions they want to agree upon by agreeing on the language that they want to use in expressing 
the conclusions that they have mutually arrived at in talking about the matters at hand that concern them. They are seeking agreement about how they should be talking and the words they should be using to make a point that is at issue or that is about a contention between them. The great mistake that they often are making is in presuming that their terms are amendable to precise definitions of essences, or essential forms of typical types of things. What they are presuming is that there are fixed natural paradigms and exemplars for the words that we or they use.

What we think we know requires that we know the language that we use to talk about the things that we think we know. We need to construct the language out of usages to talk about what has not been experienced before. Especially this is the case when we talk about what we know that turns out not to be true. To reach accord or agreement we also have to come to an agreement about how we use and interpret visual schemata that we create for expressing what we think that we know. Not only do we need to come to terms in the printed word with the way we express what we think we know, but we also have to come to terms with the uses of pictorial and mathematical representations that we use to interpret what we think we know in our ordinary ways of speaking about them.

It is this creative construction of language in science that marks the progress in the sciences. It this awareness of meaning shifts in the language of science in the manner of Ian Hacking in his work The social construction of what? That also marks the work of Evelyn Fox Keller in her work on nature-nurture debates. As she says:

Interpretations (or descriptions) are cashed out in experiments, with different interpretations leading to the design and execution of different kinds of experiments. When successful, they point the way to observations of different kinds of phenomena, which, in turn, are taken to lend credence to the interpretations that generated them in the first place. It is this sense that, even under a realist view, one can say that language 'constructs' knowledge. By simultaneously facilitating certain research trajectories, and foreclosing others, descriptions shape the construction (articulation) or the edifice we call scientific knowledge. (1992: 177)

Sometimes we need to simplify the complexity of what we are talking about to find simpler words to use or to use simpler visual and formal graphical representations to come to terms with our understanding of so much of the complexity that in our lives we for the most part encounter. In mathematics and in the sciences and especially in technology, visual representations do in a useful way simplify, or even sometimes oversimplify, much of what we think of as knowledge and truth. Such mathematical and technical models and frames are perceived as excessively complex. Most of them seem to be intuitively beyond our immediate ability to grasp their linked patterns and complete structures. But so long as we can explore the complexity of them with the rules that generate them we find our trips through them can lead to finding extraordinarily highly complex structural patterns that may be prove useful in mapping our practical applications in the use of them. 
But such formal complex models have an underside and a dark side in their uses by the various sciences and technologies when we interpret their technical language interpretations in common sense terms, especially in interpreting the rules used to generate them in ordinary language terms. What is important then to see is how visual representations can like the terms of our language produce false or oversimplified representations of what seem infinitely complex experiences. In the end what we seek is not rational knowledge in our rational linguistic and graphic frames, but knowledge of our ignorance that we are able to discover when we misrepresent our convictions by misrepresentations of measurements and orientations that we introduce in our linguistic and graphic frames in trying to warrant their scientific validity.

Just as we orient ourselves by turning our line of sight around the point on which we stand, so we equally circle in our language finding the right words to make our point. Note that the most stand pat point of orientation for any individual is looking outside from inside. The next individual stand pat point is in the orientation we have of looking straight ahead. We stand fixed on something staring at it that lies out on our horizon that is straight ahead. Such is the act of pointing. It is an act of linear extension. It is the way our noses go. 'Follow your nose. Straight ahead!' But even then we can have no knowledge of our true orientation if we have no knowledge of whatever it is that we see out there outside us that seems to be positioned in front of us straight ahead. And if something is blocking our view such as a hill, a building, or a tree, how is it possible to establish any horizon as a distant line surrounding or circling us that is lying before us straight ahead?

In standing still a horizon nevertheless is necessary to indexing or orienting ourselves to what is straight ahead. And it is looking straight ahead at a point resting on a horizon that is at the very basis of what is thought as our sense of direction when we are standing still on what is assumed to be level or flat surface. Our true orientation or heading is on that line on that presumed level plane moving toward the horizon that we face that is straight ahead. But if a line determines a plane, how do we know the plane we stand on is flat and level? It seems all too arbitrary as lines and planes mutually define each other.

But to have awareness of our orientation or our sense of direction, we also in our orientation need to make comparisons with other directions. And in looking straight ahead without looking around we have no conception of our orientation without reference to what is about us or around us, nor do we have any conception of the other things that are related to what we are directly seeing if we do not turn our head. Consequently, unless we move forward, up or down, or turn on our situated spot we have no sense of direction in reference to anything. We are static or stable on a spot which has no definite referenced location. Note in sports in track we get 'ready, set, go'. Note the multiple uses of the verbs 'set' and 'run' in our English Language Usage. The variety of contexts of their variety of uses indicates that these imperative verbs are a basic psychological frame by which we frame track experiences in running. 
Likewise from another point of view when we describe what we see from where we stand just looking straight ahead, we have no conception of what it is that is directly out there in our line of sight. Just as in radar when I stopped the directional line of the radar sweep on a blob of light straight ahead, I had no conception of what it was that was straight ahead unless the sweep of the radar continued to turn and redirect my perspective or orientation back to that seeming static blob of light. When we look ahead to see an object out there while focusing on a point, we are also focusing on our straight ahead orientation.

But note when we change our line of sight from straight ahead to the line of sight that we find when we turn our head towards the direction of our right arm, we have created a new orientation that is a quarter of a turn to the right, or as we will illustrate later that it is a clockwise motion. To do this turn is simply to follow the Drill Sergeant's command, 'Right face!' We turn to the right and then face it. Note the word face is the command, and right is a description of how to execute the command. Commands are a description of what to do and the order to do it!

Thus rotating and facing to the right is an angular rotation which enables us to measure the change of our bodily rotation. Four right turns make for a return to our original line of sight. Angular rotation is simply an alignment of parts of our body with lines of sight. Equally our standing straight and looking down gives us another angular rotation of up and down, which again are rotations that give us balance while looking straight ahead. Note how our ordinary ways of talking about seeing outside, seeing straight ahead, and about our bodily rotation, right and left, up and down help us in ordinary language interpret, and eventually technically help us, to define our orientations and our balance. The following Figure 5.1 indicates how our four turns to the right generates our sense of angular measurement based as they are upon four right turn rotation.

We use our index finger to orient ourselves either to our line of sight facing straight ahead. Or we can use our index finger to point to our right and then we turn to orient ourselves to see our pointed finger now oriented straight ahead that was once oriented to the left. We turn our attention as we turn always to the right, again and again as we keep turning rotating to whatever we want to face. Note that turning around in rotating backwards is two right turns to the right or two left turns to the left.

Note the symmetry in a complete rotation. Rotation is how we measure angles. The sum of four right turns is equal to a single rotation. Turning and pointing are the ways we organize attention on any level plane. And if we lie down on our left side pointing and facing ahead, we can then point with our right arm straight up. We have entered into three dimensions simply by rotating from a stand point either standing or lying on the ground. And if we stand up again after lying down, we can again look up and down, either up and down from our perspective of looking straight ahead. Note we have difficulty looking up when standing up. We get dizzy looking up at sky scrapers. 


\section{Angular Rotation}

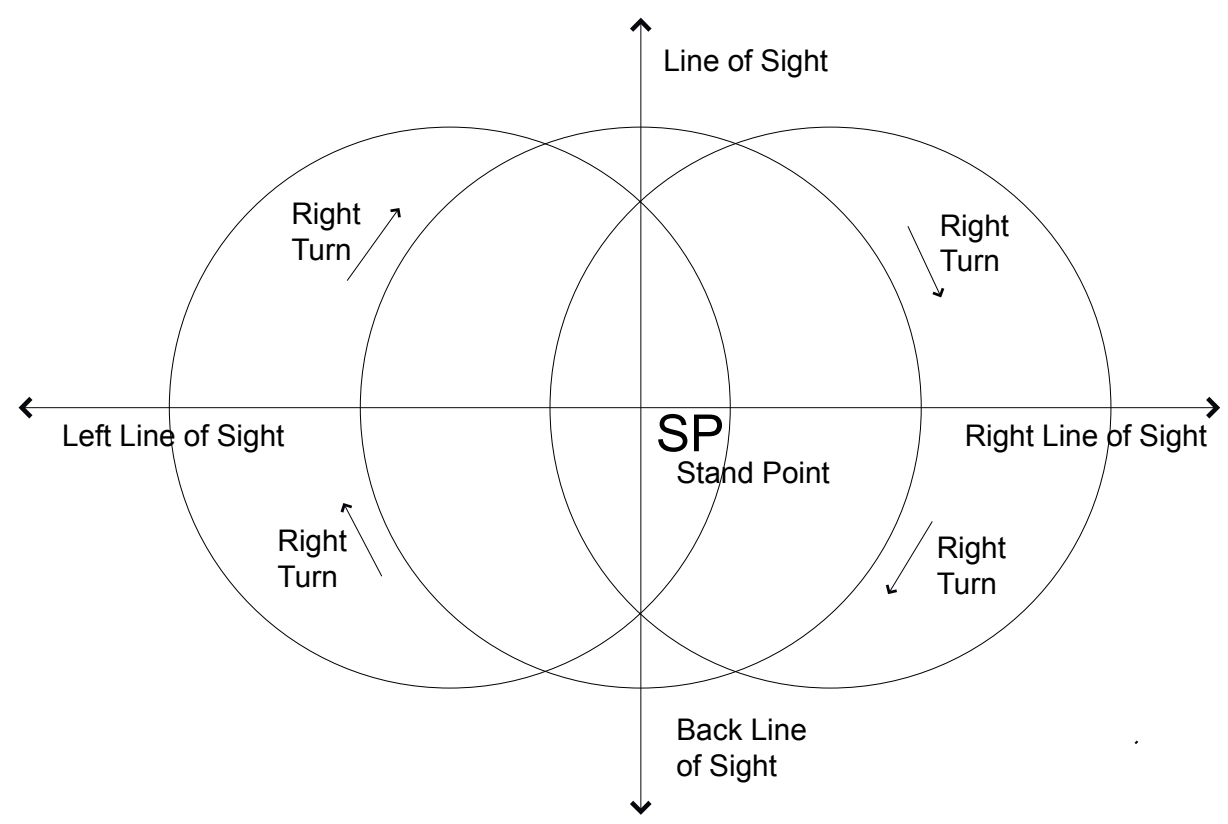

Figure 5.1: Angular rotation

Thus, pointing and turning to our right either lying on our side or standing up frames the whole sphere of our direct attention. But note though we can point and aim and turn around in three directions straight ahead, to the right, and up, we have no biological sense of where we are in space accept for three factors 1) our sense of weight, 2) our balance, and 3) our horizon. We have only a sensuous frame of reference, but no direct indication or any sense of our location. Note that this way of talking about orientation is embedded in the ordinary language that we speak.

But note that this way of speaking can change for those who live at the foot of a mountain who have a mountain as a point of orientation on the horizon. The mountain becomes a point of reference as we turn and rotate in reference to it. How easy it is to know your orientation when you live directly south from a mountain with a sharp peak as a point of reference to determine what is north. It has always been difficult for me to sense the direction of the compass points living in a valley where a mountain has no precise compass direction to be able to orient ourselves in using it. I found this to be the case in State College, Pennsylvania, when I was in school there in the service during World War II. I never quite knew the direction of Mt. Nitany that stands and splits two valleys that merge into one valley at State College. Roughly in my mind the 
valleys were running towards the southeast. But I had no precise conception of their compass direction.

But note there are other ways of speaking of orientation depending on the language we speak. In aerial gunnery there was a clock orientation language. Planes could be coming in twelve o'clock high or low, three o'clock, or three o'clock high. 'Nine o'clock low, and six o'clock high.' Another language of orientation can be determined by directions of north, east, south, and west, which again like right and left and straight ahead can be the language of a different sort of compass. Note again as previously mentioned that farms in the Midwest were laid out with surveys that drew lines based upon compass headings at the time which varied with local magnetic variations, so what was said to be north was not true north as measured by the directions of the fixed stars that could be determined by the rotation of the celestial sphere.

Thus the fundamental stance we take on orientation depends on the ordinary language meaning for direction that has developed in different contexts depending on the evolution of our language and the location used in talking about orientation. Location always requires a standardized point of reference that is politically defined to determine how we are to conceive angles and distance. In navigation we needed a range and bearing to determine our location relative to an agreed upon fixed external location. Location is always relative to units of angular measurement and to units of length and relative to a precisely agreed upon standardized points of location. But what we point to must be in our line of sight, so what we always see is always bound by what we think of as objects. But again objects are usually described in the language of ordinary usage. When we name specifically defined objects the language we use to name those objects finds its way into the language of our ordinary discourse.

It is this same sort of orientation and focus that we find when we search for words to help us eventually find different words that we need to express any point or schematic representation of order or structure that we want to make. But the same circling we find again in moving through synonyms. We are again shifting perspectives or orientations. It is in a comparable way a ring of synonyms. It is just circling around looking for a term that is expressing our fundamental stand and orientation on the point that we are trying to make.

Consequently all communication is dual, first finding words to say what we want to say and second using those words to say what it is that we want to say. We circle through meanings and definitions, and we find in those word meanings so as to communicate in a grammatical or propositional fashion that can be schematized into a logical order. Communication thus in its beginnings is circling and searching for terms that will help us say what we want to say in an orderly and schematic fashion within a defined reference grid that defines our orientation. Communication starts with defining what it is that we want to say within that orderly oriented vernacular frame that is derived and interpreted from our personal language that we use to tell others where we stand. 
What makes us think that definitions are linear and not circular is that the logical terms in definitions appear in our linguistic expressions are propositional, that is, their grammar is sentential. The fact of the matter is that in interpreting terms we are relating terms that are involved in the definition of what we want to say in a propositional or linear order. But finding words in speaking and writing for what we want to say we need to communicate substantive content. We need to precise the interpretations of the words we use or the terms we use in propositions that we want to reason with. We need to put them into statements or sentences that clarify what we want those words to mean for our listener or reader. Our preparing to do so always involves a preliminary circular activity searching through each other's vocabulary to adjust our orientations to what is said.

In sum communication thus always has a dual purpose:

- To clarify what we are saying to our audience,

- And after an agreed upon clarification to state or make a point propositionally about how we want to relate to that audience in a cooperative manner in getting things done. 